The method has, however, an interest, as showing that there is a way of drawing any given case; and the variety of methods of expressing particular functions that have already been discovered renders it in the highest degree probable that in every case a simpler method can be found. Theire is still, therefore, a wide field open to the mathenatical artist to discover the simplest linkworks that will describe particular curves.

The extension of this demonstration to curves of double curvature and surfaces clearly involves no difficulty.

Further Note on the Motion of a Plane under certain Oonditions. By SAMUEL ROBERTS.

[Read June 9th, 1876.]

IN my paper "On the Motion of a Plane under certain Conditions" (Proc., Vol. III., p. 286 et seq.), the movement was determined by the paths of two of the points of the plane over a coincident stationary. plane. I propose now to consider briefly two other elementary cases to which I referred at the close of my former paper, and relative to which I bad then obtained some general results.

Those cases are: (1) when the motion is determined by the path of a point of the moving plane and by the envelope of one of its lines; (2) when the motion is determined by the envelopes of two of the lines of the plane.

(A.) Line-directrix and Point-directrix.

(1.) Locus of Points.

In the first case the problem is to determine the characteristics of the locus of a point $P$ carried by a constant angle $\triangle B C$ moving in its plane, so that while the side $A B$ always touches a fixed curve, which I call the line-directrix, the other side moves with a fixed point $C$ always on another fixed curve, which I call the point-directrix. The two direcirices are supposed to lie in the same plane as the moving angle.

I consider in the first instance a simple case, namely, when the linedirectrix is a point, and the point-directrix is a straight line.

The locus is a circular curve of the fourth order and sixth class, having usually two finite double points and another at infinity, where the point-directrix meets the line at infinity. The line-directrix is the focus formed by the circular asymptotes.

If, however, the apex $B$ moves on the point-directrix, then the locus 
of a point on $A B$ (produced as may be necessary) is a conchoid of Nicomedes, and one finite node passes to infinity and joins there the other infinitely distant node. The same takes place for all points on a line through $\mathrm{C}$ parallel to AB. . (Proc., Vol. II., p. 128.)

- Suppose now that we take $n$ points for our line-directrix and $m^{\prime}$ lines for our point-directrix, the order of the componnd locus is $4 \mathrm{~nm}^{\prime}$ and its class is $6 \mathrm{~nm}^{\prime}$.

The corrections necessary to obtain the general order and class have to be investigated.

If a multiple point of the order $p$ exists on the point-directrix at $C$, it follows from the nature of the description that the corresponding position of $P$, the describing point, determines on the locus a multiple point of the order $p$. There are $2 n$ such positions of $\mathrm{P}$ corresponding to $2 n$ tangents common to the line-directrix and a circle about $C$ as centre. That is to say, the multiple point $C$ determines $2 n$ multiple points on the locus. Again, if there is a tangent to the line-directrix touching it at $p$ points, there are $2 \mathrm{~m}^{\prime}$ positions of $\mathbf{P}$ corresponding to this multiple tangent, and each position determines a multiple point of the order $p$.

It may be inferred also from the nature of the description that these multiple points have the same number of multiple tangents that the corresponding multiple points on the point-directrix have of coincident tangents, or the corresponding multiple tangents of the line-directrix have of coincident contacts, as the case may be. In short, the singalarities produced are similar individually to those prodacing them. Unless there are special relations existing in the construction, a case which is now excluded, these are the only singularities produced by singularities of the directrices. With regard then to our special directrices, we have to correct for $\frac{n(n-1)}{2}$ double tangents passing through the points of the line-directrix taken two and two, and for $\frac{m^{\prime}\left(m^{\prime}-1\right)}{2}$ intersections of the $m^{\prime}$ lines of the point-directrix; but, if further we suppose that there remain $r$ double tangents, c iuflexions on the linedirectrix, and $\delta^{\prime}$ nodes, $\kappa^{\prime}$ cusps on the point-directrix, we must correct by deduction.

Thus we obtain for the general directrices:

$$
\begin{aligned}
& \text { Order of locus } \quad 4 m n^{\prime} \text {, } \\
& \text { class of locus } 6 n m^{\prime}+2 n(n-1) n^{\prime}+2 m^{\prime}\left(m^{\prime}-1\right) n \\
& -4 \tau m^{\prime}-6 m^{\prime}-4 \delta^{\prime} n-6 x^{\prime} n \text {, } \\
& \text { or } \quad 6 n n^{\prime}+2 m m^{\prime}+2 n n^{\prime} \text {. }
\end{aligned}
$$

The only cusps are those introduced by the inflexions and cusps of the linc and point-directrices respectively in number

$$
2 \iota m^{\prime}+\underline{a} x^{\prime} n \text {. }
$$


The circular points are multiple in the order $n m^{\prime}$; the $m^{\prime}$ points, in which the point-directrix meets the line at infinity, are multiple in the order $2 n$.

It must be always remembered that special positions of $P$ relative to the traversing angle may occasion an alteration of the singularities. So also special relations of the angle to the directrices, or of the directrices to each other, are excladed from cousideration.

Even in the general case, the multiple points not due to singularities on the directrices are of a complex character. For instance, it has been remarked that, for a point as line-directrix and a line as pointdirectrix, the circular asymptotes intersect in the line-directrix, in other words, in the focus of the line-directrix. But the point singularities at infinity are not in general affected by finite singularities of the directrices. Hence the multiple circular points have coincident tangents in consequence of contiguous multiple points. There are $n$ real foci of the line-directrix. Through ench of these will pass $m^{\prime}$ lines meeting the locus at a circular point in $n m^{\prime}+m^{\prime}$ consecutive points. In fact the $n m i$-fold circular point is accompanied by $n$ contiguous points multiple in the order $m^{\prime}$.

In like manner, the loci of points on a line through $\mathrm{C}$ parallel to $\mathrm{BA}$ will have, at the points where the point-directrix meets the line at infinity, $m^{\prime}$ pairs of contiguous multiple points of the order $2 n$.

It is useful to regard the motion in different points of view. For instance, if wo draw a line through $P$ parallel to $B A$ and join $P C$, the locus is evidently the same as that of the apex of a constant angle, one side of which touches a parallel of the original directrix, while the other moves with a fixed point on the point-directrix. Again, if we draw a line through $\mathrm{C}$ parallel to $\mathrm{BA}$, it appears that the same motion is produced by making the apex of the angle move along the pointdirectrix, while one side envelopes a parallel of the original linedirectrix.

The locus of a point in BA (continued if necessary) is the same as that of the apex of a constant angle moving in the same manner relative to the directrices.

The question suggests itself, whether the reduction of the gross order, which must take place when we substitute a parallel of the linedirectrix for the line-directrix itself, arises from the singularities of the parallel or otherwise. It appears that this effect is produced by the breaking up of the locus into line components, one of which is the locus required, the other corrcsponding to a different line $\mathrm{BA}$.

To shew this, let the line-directrix be such that its parallel breaks up as indicated by the tangential equation

$$
\left(a^{2}+\beta^{2}\right) \phi^{2}+\psi^{2}=0
$$

where $\phi, \psi$ are functions of the order $n-1$ and $n$ respectively iu $\alpha, \beta$. 
It will suffice to suppose that the moving angle is a right angle, so that we have, for three of the conditions, say,

$$
\alpha x+\beta y-1=0, \quad \beta\left(x-x^{\prime}\right)-\alpha\left(y-y^{\prime}\right)=0, \quad\left(x-x^{\prime}\right)^{2}+\left(y-x^{\prime}\right)^{2}=l^{2},
$$

giving $a^{2}+\beta^{2}=\frac{\left(a x^{\prime}+\beta y^{\prime}-1\right)^{2}}{l^{2}}$, and (a) breaks up into two conditions. Hence, if the line-directrix is such that its parallel breaks up, the locus of the apex of an angle or other point rigidly connected with it also breaks up.

But the parallel of the parallel of a curve necessarily breaks up. Therefore the gross order $4.2 n . m^{\prime}$ must be divided by 2 . Hence also we see that the multiple circular points on the line-directrix do not reduce the order, when at lenst the point-directrix is not circular.

If, in like manner, we take the class with reference to the parallel of the original line-directrix, we have

$$
6.2 n m^{\prime}+2(2 n+2 m) m^{\prime}+2.2 n n^{\prime} ;
$$

and the class of one component is

$$
6 n m^{\prime}+2 m m^{\prime}+2 n n^{\prime}+2 n m^{\prime},
$$

to be diminished, as I shall presently shew, by $2 \mathrm{~nm}^{\prime}$, on account of multiple circular points.

\section{Oircular Directrices.}

If we take a point as the line-directrix and a circle as the pointdirectrix, the locus of a point carried by the angle is of the sixth order, the circular points are triple points, each having a pair of coincident tangents which intersect the conjugate pair at the centre of the fixed circle. There are three other nodes. The remaining tangents at the triple points intersect in the fixed point, and the two singular foci and the nodes lie in a circle. This is a case of the three-bar motion curve in which two of the three singular foci coincide, and the actual description by means of three links is impracticable.

The equation may be obtained as follows:-

Let $\mathrm{ABC}$ be the angle whose tangent is a constant $t$, and which moves so that $\mathrm{BA}$ passes through the origin of coordinates while $\mathrm{C}$ moves on a circle whose equation is $(x-a)^{2}+y^{2}=r^{2}$.

Let $\mathrm{P}$ be the describing point, and draw $\mathrm{PD}$ perpendicular to $\mathrm{BC}$. Then, if $\mathrm{PD}=l, \mathrm{CD}=p, \mathrm{BD}=q$, and $\mathrm{BC}$ makes the angle $\phi$ with the axis of $x$, and if $x_{1}, y_{1}$ are the coordinates of $\mathrm{B}, x_{2}, y_{9}$ those of $\mathrm{C}$, and $X, Y$ those of $P$, we have

$$
\begin{array}{ll}
y_{1}=\frac{\sin \phi-t \cos \phi}{\cos \phi+t \sin \phi} x_{1}, & \left(x_{2}-a\right)^{2}+y_{2}^{2}=r^{2}, \\
x_{1}=\mathrm{X}-l \sin \phi-q \cos \phi, & x_{2}=\mathrm{X}-l \sin \phi-p \cos \phi, \\
y_{1}=\mathrm{Y}+l \cos \phi-q \sin \phi, & y_{2}=\mathrm{Y}+l \cos \phi-p \sin \phi,
\end{array}
$$


from which we get

$$
\begin{aligned}
&(\mathrm{X}-\mathrm{Y} t) \sin \phi-(\mathrm{Y}+\dot{\mathrm{X}} t) \cos \phi+q t-l=0, \\
&\{p \mathrm{Y}+l(\mathrm{X}-a)\} \sin \phi+\{p(\mathrm{X}-a)-l \mathrm{Y}\} \cos \phi \\
&+\frac{r^{2}-l^{2}-p^{9}-(x-a)^{9}-y^{2}}{2}=0 ;
\end{aligned}
$$

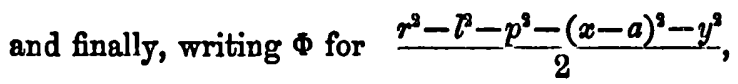

$$
\begin{aligned}
& \{(\mathrm{Y}+\mathrm{X} t) \Phi+(q t-l)[p(\mathrm{X}-a)-l \mathrm{Y}]\}^{2} \\
& +\{(\mathrm{X}-\mathrm{Y} t) \Phi-(q t-l)[p \mathrm{Y}+l(\mathrm{X}-a)]\}^{2} \\
& \quad-\left\{(p+l t)\left(\mathrm{X}^{2}+\mathrm{Y}^{2}\right)-a l(\mathrm{X} t+\mathrm{Y})-a p(\mathrm{X}-\mathrm{Y} t)\right\}^{2}=0 .
\end{aligned}
$$

Writing this in the form

$$
[\mathrm{L} \Phi+(q t-l) \mathrm{M}]^{2}+\{\mathbf{P} \Phi-(q t-l) \mathrm{Q}\}^{2}-\{\mathrm{LQ}+\mathrm{MP}\}^{2}=0,
$$

we see that the circular points are triple, and the three usually finite nodes lie on a circle through the origin and the centre of the fixed circle.

To find the tangents at the circular points, we may substitute $a$ for $\frac{X+i Y}{2}, \beta$ for $\frac{X-i Y}{2}$, then the coefficient of $\beta^{3}$ is the same as in

$$
\left(1+t^{2}\right)\left(\mathrm{X}^{2}+\mathrm{Y}^{2}\right) \Phi^{2}+\left[k\left(\mathrm{X}^{2}+\mathrm{Y}^{2}\right)+l_{1}\right] \Phi+\left(m \mathrm{X}^{2}+\mathrm{Y}^{2}+n_{1}\right)=0 .
$$

where $k$ and $m$ are constant, and $l_{1}, n_{1}$ are of the first order in $\mathrm{X}, \mathrm{Y}$. We have for the tangents

$$
a(2 a-a)^{2}=0, \text { or }(X+i \mathrm{Y})[(\mathrm{X}-a)+i \mathrm{Y}]^{2}=0,
$$

shewing that there are two pairs of coincident tangents intersecting at the centre of the fixed circle, and that the remaining pairs intersect at the origin.

Again, if we take a circle as line-directrix and another circle as pointdirectrix, we have a locus which breaks up into two curves of the same nature as the one just disctissed.

If we take as line-directrix a circle and as point-directrix a straight line, the locus consists of two curves of the fourth order and sixth class, of the sane kind as when we take a point and line as directrices. Although the loci break up, we are obliged to take the components into account, because the locus does not break $n p$ in the first of these elementary cases.

When, therefore, the line-directrix consists of $n-2 p$ points and $p$ circles, and the point-directrix consists of $m^{\prime}-y$ lines and $q$ circles, the order of the general locus is

or

$$
4(n-2 p)\left(m^{\prime}-2 q\right)+6(n-2 p) q+8\left(m^{\prime}-2 q\right) p+12 p q,
$$

and no correction is necessary on account of the breaking np of the directrices. 
Again, for the class we have

$$
6(n-2 p)\left(n^{\prime}-2 q\right)+10(n-2 p) q+12\left(m^{\prime}-2 q\right) p+20 p q,
$$

and we must add the correction for nodes and double tangents (leaving however the two multiple circular points of the point-directrix),

$$
\begin{aligned}
& 4 m^{\prime} \cdot \frac{n-2 p \cdot n-2 p-1}{2}+4 n \frac{m^{\prime}-2 q \cdot m^{\prime}-2 q-1}{2} \\
+ & 4 m^{\prime} \cdot 2 p(n-2 p)+4 n \cdot 2 q\left(m^{\prime}-2 q\right)-4 n q(q-1) \\
+ & 4 m^{\prime} \cdot 2 p(p-1)+4 n \cdot 2 q(q-1) .
\end{aligned}
$$

And if there are $r$ double tangents and 1 inflexions on the linedirectrix and $\tilde{o}$ double points, $x^{\prime}$ cusps on the point-directrix, we have over corrected, and must now deduct

$$
4 m^{\prime} r+6 m^{\prime} \iota+4\left[\delta^{2}-q(q-1)\right] n+6 n x^{\prime} .
$$

so that finally, for the general class, we have

$$
6 n m^{\prime}+2 m m^{\prime}+2 n^{\prime} n-6 n q-4 m^{\prime} p .
$$

The circular points are multiple in the order $n m^{\prime}+n q$, and the $m^{\prime}-2 q$ points at infinity not circular of the point-directrix are of the order $2 n$.

There are at the circular points $q$ groups of $2 n$ coincident tangents intersecting their conjugates at the $q^{2}$ singular foci of the point-directrix, and $(n-2 p)$ groups of $\left(m^{\prime}-q\right)$ coincident tangents intersecting at the $(n-2 p)^{2}$ ordinary foci of the line-directrix, and $p$ groups of $2\left(m^{\prime}-q\right)$ coincident tangents intersecting their conjugates at the $p^{2}$ singulur foci of the line-directrix.

These coincident tangents are not altogether cuspidal, but are in great part due to adjacent multiple parts.

There remains the equivalent of $2 n q$ cusps. We have further the $2 \imath m^{\prime}+2 x^{\prime} n$ cusps introduced by singularities of the directrices.

\section{The line-directrix touches the line at infinity.}

When the line-directrix tonches the line at infinity, there is a reduction of order and class.

For, take as line-directrix $n-p$ finite points and $p$ at infinity, and as point-directrix $m^{\prime}$ lines. Then the order of the general locus is

$$
4(n-p) m^{\prime}+2 p m^{\prime}=(4 n-2 p) m^{\prime} \text {, }
$$

and no correction is necessary.

For the class we have, correcting as before,

$$
\begin{aligned}
6(n-p) m^{\prime}+2[m(m-1)-p(p-1)] m^{\prime}+2 m^{\prime}\left(m^{\prime}-1\right) m \\
-4\left(r-\frac{p(p-1)}{2}\right) m^{\prime}-6 m^{\prime}-4 \delta^{\prime} n-6 x^{\prime} n .
\end{aligned}
$$

giving

$$
6(n-p) n i^{\prime}+2 m n^{\prime}+2 n^{\prime} n \text {. }
$$

The modifications in the multiple points at infinity may be traced as 
before. The circular points are of the $\operatorname{order}(n-p) m^{\prime}$ and the $m^{\prime}$ points at infinity of the point-directrix are still of the order $2 n$. There are at a circular point $n-p$ groups of $m^{\prime}$ coincident tangents intersecting their conjugates in the $(n-p)^{2}$ foci of the line-directrix. For simplicity's sake, I have treated the sereral cases successively. We might have combined them with no great difficulty in accordauce with the hypothesis that the directrices are both circular and parabolic.

\section{(2.) Envelopes of Lines.}

There is in general a one-to-one correspondence between the points of the locus and the lines of the envelope. When the line-directrix is a point and the point-directrix is a line, the envelope of a line, taken generally, is of the fourth class and sixth order, having four double points and six cusps, and being therefore unicursal. It is also circular. Hence, if we take $n$ points for the line-directrix and $m^{\prime}$ lines for the point-directrix, the class is $4 n^{\prime} m$, which holds good generally. When a multiple point is produced in the locus of a point on the traversing line by a singularity of the directrices, a corresponding multiple tangent is given to the envelope. Hence, for the general order we get, by correcting as previously,

$$
6 n m^{\prime}+2 m m^{\prime}+2 n n^{\prime} \text {. }
$$

And we may trace the nature of the singularities at infinity to some extent. Thus there is a multiple tangent of the order $2 \mathrm{~nm}^{\prime}$, or, at least, we have its equivalent while the circular parts are multiple in the order $n m^{\prime}$.

The circumstances of the general case are, however, more readily comprehended when we consider the envelope of a line passing throngh C, while $B$ coincides with $\mathbf{C}$, so that the apex moves on the pointdirectrix. When we take a point and line for directrices, as before, the envelope of the line through $\mathbf{C}$ is a parabola. This gives us for the corresponding class in the more general case $2 \mathrm{~nm}^{\prime}$, and for the order $2 n m^{\prime}+n m^{\prime}+n m^{\prime}$. And the envelope of a line taken generally is the parallel of such an envelope for a line through $\mathrm{C}$; that is to say, it is of the class $4 n m^{\prime}$, and the order

$$
4 n m^{\prime}+2\left(2 n m^{\prime}+m m^{\prime}+n^{\prime} n\right)-2 n m^{\prime}=6 n m^{\prime}+2 m m^{\prime}+2 n n^{\prime},
$$

the number $2 n m^{\prime}$ being deducted from the gross order in consequence of the tangencies at infinity. The characteristics of the general envelope are therefore known by the theor'y of parallels. The circular points are of the order $n m^{\prime}$, and there are $n m^{\prime}$ contiguous pairs of nodes at infinity, accounting in this case for the $2 \mathrm{~nm}^{\prime}$-fold tangent. The other $n m^{\prime}+n^{\prime} m$ points at infinity are double.

If, in the general case, we take the envelope of a line through $\mathbf{C}$, it is still of the class $4 \mathrm{~nm}^{\prime}$, \&c. ; but it is of such a form that its parallel 
breaks up, and the two components correspond to different lines of the plane.

. If the line-directrix touches the line at infinity, we have reductions corresponding to those of loci in the like case; namely, the class is $(4 n-2 p) m^{\prime}$, and the order $6(n-p) m^{\prime}+2 m m^{\prime}+2 n^{\prime} n$. In the special case when $B$ and $C$ coincide and the line passes through $C$, the class is $(2 n-p) m^{\prime}$, and the order $2(n-p) m^{\prime}+m m^{\prime}+n^{\prime} n$.

I suppose that we might obtain the values of the order and class of envelopes in the case of circular directrices by the method used in the case of loci.

\section{(B.) Two Line-Directrices.}

(1.) Locus of Points.

The second case mentioned at the beginning of this paper requires us to determine the characteristics of the locus of a point carried in its plane by a constant angle whose sides touch two directrices.

It will be shorter to take at once the circular directrices, for which purpuse we bave to consider the crse of two points as directrices. The locus of the apex is here a circle; that of any other point is a limaçon. Next we will take a point and a circle as directrices. The locus of the apex is a limaçon; that of a point not the apex consists of two limaçons. Again, if the directrices are two circles, the locus of the apex consists of two limaçons; that of a point not the apex consists of four limaçons.

Consider, then, two directrices consisting respectively of $n-2 p$ points, $p$ circles; and $n^{\prime}-2 q$ points, and $q$ circles.

The order of the locus described by the apex is

$$
2(n-2 p)\left(n^{\prime}-2 q\right)+4(n-2 p) \dot{q}+4\left(n^{\prime}-2 q\right) p+8 p q=2 n n^{\prime},
$$

which requires no correction in the general case.

For the class we have

$$
2(n-2 p)\left(n^{\prime}-2 q\right)+4(n-2 p) q+4\left(n^{\prime}-2 q\right) p+8 p q=2 n n^{\prime} ;
$$

and the correction is, if we continue the notation previously used,

$$
\begin{aligned}
& \frac{2 n-2 p \cdot n-2 p-1}{n}+\frac{2 n^{\prime}-2 q \cdot n^{\prime}-2 q-1}{2} \\
& \quad+4 \quad-1) n^{\prime}+4 q(q-1) n \\
& \quad+4(n-2 p) p \imath^{\prime}+4\left(n^{\prime}-2 q\right) q u \\
& \quad-2 \pi n^{\prime}-3 \iota n^{\prime}-2 \tau^{\prime} n-3 \imath^{\prime} n ;
\end{aligned}
$$

and the general class is $2 n n^{\prime}+n^{\prime} m+n m^{\prime}$.

The circular points are multiple in the order $n n^{\prime}$, and there are cuspidal coincident tangents as well as tangents otherwiso coincident. 
If the describing point is not at the apex, the class and order are doubled. The circular points are also cuspidal in a higher degree.

If the directrices touch the line at infinity, there are further reductions. Thus, if the directrices touch that line $r$ and 8 times respectively, we have for the order of the apex locus

$$
2(n-r)\left(n^{\prime}-8\right)+r\left(n^{\prime}-8\right)+8(n-r)=2 n n^{\prime}-m n^{\prime}-8 n ;
$$

and the class is

$$
2(n-r)\left(n^{\prime}-s\right)+\left(n^{\prime}-8\right) m+(n-r) m^{\prime} .
$$

We may evidently consider the locus of a general point as described by the apex of a constant angle moving about two parallels of the directrices. But, in order to make use of this apparently more simple conception, we have to take account of the singularities of the parallels, particularly at infinity. If two directrices are such that their parallels break up, the locus of the apex breaks ap.

\section{(2.) Envelopes of Lines.}

For two points as directrices the envelope of a line through the apex is a point on the circle described by the apex.

By taking a system of $n$ points for ono line-directrix, and $n^{\prime}$ points for the other, we get for the class generally $n n^{\prime}$.

The order is the correction

or

$$
\begin{gathered}
\frac{2 n^{\prime}\left(n^{\prime}-1\right)}{2} n^{\prime}+\frac{2 n(n-1)}{2} n^{\prime} \\
-2 r n^{\prime}-3 \imath n^{\prime}-2 \tau^{\prime} n-3 \iota^{\prime} n, \\
n m^{\prime}+n^{\prime} m .
\end{gathered}
$$

For a line not passing through the apex we must have the parallel of a corresponding envelope by a line through the apex.

The class is $2 n n^{\prime}$, and the order

$$
2 n n^{\prime}+2 n m^{\prime}+2 n^{\prime} m \text {. }
$$

The circular points are now multiple in the order $n n^{\prime}$, and the $n m^{\prime}+n^{\prime} m$ points, in which the carre enveloped by a parallel line through the apex meets the line at infinity, are double.

If the directrices touch the line at infinity $r$ and $s$ times respectively, the class of the apex envelope becomes

$$
(n-r)\left(n^{\prime}-8\right)+r\left(n^{\prime}-8\right)+8(n-r)=n n^{\prime}-r 8,
$$

and the order is

$$
(n-r) m^{\prime}+\left(n^{\prime}-s\right) m \text {. }
$$

I infer from these formulø, what I dare say is otherwise known, that the oblique pedal of a curve is the ordinary pedal of a curve of the same order and class.

I bave not discussed some important modifications and special rela- 
tions, as, for instance, the coincidence of directrices. Special relations between the directrices as common foci, \&c. augment in a notable manner the difficulty of determining the characteristics. But such relations exist in full force when there is only one directrix, and I am not able to give satisfactory results.

In the first case, the order of the general locus is not changed when the directrices coincide; that is, it is $4 \mathrm{~nm}$. In the second case, it is $4 n(n-1)$ for a general point, $2 n(n-1)$ for the apex ; but if the angle is a right angle, the latter locus is of the order $n(n-1)$, and I presume the first order is also reduced. In these instances it is the class which offers considerable difficulty.

Notes on the Communication entitled "On the Transformation of Elliptic Functions." By Prof. W. K. Curpford, M.A., F.R.S.

Some of the following notes* would have been incorporated in the paper by the process of revision for the press, if that had not been kindly performed for me during an enforced absence from the English climate. As regards all but one of them, I am glad of the opportunity which has thus been afforded me of extension and correction. But it is a matter of great regret to me that I discovered too late the priority of $M$. Darboux in the principal theorem of the second part of the paper ; viz., the porismatic character of a polygram circumscribed to a conic and totally inscribed in a curve of order one less than the number of sides. In one of the notes to a book which it is almost inexcusable in a geometer not to have read, marked, learned, and inwardly digested, $f$ M. Darboux has stated and proved the theorem, and has followed it by further investigations of the highest interest and importance. The method even of my investigation is the same as that of M. Darboux (as indeed was inevitable from the nature of the subject), namely, the representation of a point in a plane by means of the parameters of the tangents drawn from it to a fixed conic. It is not the first time that I have had the honour of following, however distantly, in the footsteps of that eminent geometer; but on other occasions. it has been my good fortune to discover the fact in time.

\footnotetext{
- These remarks apply also to certain developments which I have since thought it better to communicate under separato titles.

+ "Sur une classe remarquable de courbes et de surfaces algébriques." Paris, Gauthier-Villars, 1873. Noto II., p. 183.
} 